THEORIA ET HISTORIA SCIENTIARUM, VOL. VI, N 1

Ed. Nicolas Copernicus University 2001

Earl R. Mac Cormac

\title{
The metaphoric brain: mind and consciousness
}

\section{Introduction}

How mind and consciousness emerge from brain processes remains one of the major scientific challenges of the twenty-first century. This problem also serves as an intersection point between the sciences and humanities. Philosophers have for centuries investigated the nature of mind and many today deny the existence of disembodied thought. Instead, they emphatically locate mind and consciousness in brain functions. Psychologists have also investigated mental activities and have recently joined neuroscientists and philosophers to form a new field, cognitive science, concerned with solving this puzzle combining techniques from these different disciplines. This interdisciplinary approach, however, has not yet come close to solving the problem but has drawn upon insights from these various disciplines to propose speculative explanatory theories. And the hypothetical nature of each theory rests upon a basic underlying metaphor. The first part of this paper will present a sampling of these metaphoric theoretical proposals demonstrating the vibrancy of each.

In the second part of the paper, I shall propose the application of the mathematics of chaos and fractals to the images of brain processes scanned during cognitive activations as a promising route to the solution of this massive problem of how to demonstrate the emergence of mind and consciousness. Both thinking and awareness are processes that occur in no one location in the brain but rather as a series of clustered neuronal activations best described by nonlinear algorithms of chaos theory with the stable attractor points as fractals. 


\section{Metaphoric descriptions of consciousness}

Metaphors are conceptual devices usually expressed in words that juxtapose the known with the unknown (Mac Cormac, 1985,1989). This juxtaposition depends upon some similarity of features between the two parts of the metaphor. If there were no such likeness, then the unkown part would remain completely mysterious. Metaphors with a great deal of similarity between the parts can be called epiphors while those with a great deal of dissimularity can be called diaphors. Metaphors are used to suggest new ways of looking at things or new ways of expressing feelings. Some metaphors become so successful in their expressions of likeness, that they become commonly accepted and are accepted as ordinary language. As these metaphors change their status, they often add new meanings to the semantic meanings of the words that they use. Metaphors are a major instrument for changes in meaning. Some diaphors become epiphors and some epiphors become ordinary language. But some metaphors are also rejected and die.

Metaphors are used not only to convey new speculative ideas, but also as the conceptual foundation stones upon which to construct an entire theory. We shall designate these underlying metaphors as basic metaphors. Although basic metaphors can be more or less speculative, like all metaphors they are true to a degree. One should not judge metaphors as only either true or false. Like fuzzy logic, they can have partial membership in both the sets of truth and falsity without worry about contradiction. Indeed, that is one of the beauties of metaphors, they are not only vibrant in their striking newness, they are not only suggestive of new possibilities, but they also express concepts which are at least minimally partially true and capable of increasing that degree of truth as further evidence appears. Hence, each of the following theories about consciousness founded upon a basic conceptual metaphor, do express a degree of truth. Yet they remain incomplete and unconfirmed.

Bernard Baars in his A Cognitive Theory of Consciousness presents an explanatory account of consciousness based upon a basic metaphor called the 'Global Workspace' (GW) (Baars 1995) and in his more recent In The Theater of Consciousness he extends and transforms the earlier metaphor of the Global Workspace into a 'Theater' (Baars 1997). Let us first consider the Global Workspace and then turn to the Theater. In the first work, Baars offers an operational definition where consciousness consists of

the set of events that can be reported with verifiable accuracy and are claimed to be conscious under optimal reporting conditions (1.2.1). It includes qualitative contents such as percepts, mental images, inner speech, and feelings of pleasure, pain, and 
affect; as well as nonqualitative contents such a currently accessible concepts, beliefs, intentions, and expectations (1.2.5) (Baars 1995:372)

These mental representations of consciousness are broadcast to the rest of the central nervous system by the Global Workspace.

In essence, we develop only a single theoretical metaphor: a publicity metaphor of consciousness, suggesting that there is a "global workspace" system underlying conscious experience. The global workspace is the publicity organ of the nervous system; its contents, which correspond roughly to conscious experience, are distributed widely throughout the system. This makes sense if we think of the brain as a vast collection of specialized automatic processors, some nested and organized within other processors. Processors can compete or cooperate to gain access to the global workspace underlying consciousness, enabling them to send global messages to any other interested systems. Any conscious experience emerges from cooperation and competition between many different input processors (Baars 1995:xx).

Baar then develops seven models of GW with each succeeding model encompassing more and more empirical and psychological evidence. Three major parts, however, compose the core of GW theory. They are: (1) the global workspace itself which Baars likens to a bulletin board and a radio station; (2) Unconscious specialized processor found within the brain; and (3) unconscious Contexts found within and influenced by culture. The conscious contents of GW include Attention, Short Term Memory and Working Memory. Long Term Memory is found in the parallel distributed processor of Unconscious specialized processors. Activated Memory is found in the Contexts. But central to Contexts is a hierarchy of goals. Within consciousness, a single goal is chosen and knowledge sources are integrated into a single coherent experience thereby changing by addition the Contexts.

Baars suggests that his GW theory has origins in brain processes, specifically the extended reticular-thalamic activity system (ERTAS).

We can therefore suggest that the ERTAS underlies the "global broadcasting" function of consciousness, while a selected perceptual "processor" in the cortex supplied the particular contents of consciousness to be broadcast. (These are typically perceptual contents, because the ERTAS receives collateral pathways from all sensory tracts; and of course, we have previously remarked on the favored relationship between conscious experience and perception). These conscious contents, in turn, when they are broadcast, can trigger motoric, memory, and associative activities (Baars 1995: 126).

By locating the GW theory in ERTAS, Baars suggests that neuronal activity in the brain forms the cause of mental activity. And in doing so he avoids (perhaps ignores) the question of how conscious mental activity like biofeedback can 
affect bodily, physical activity. He also sidesteps the philosophical problem of whether the categories of physical and mental have the same ontological basis.

Baars demonstrates a self awareness of his use of metaphor. From time to time in his odyssey of developing seven GW models, he takes time out to reflect upon how far his metaphoric usage has taken him down the path of explanation. After developing two models, he states:

Has the "publicity metaphor" helped to clarify the issues so far? Consider how the GW model resembles a publicity organ like a newspaper. First, a global workspace can distribute a message to a wide public of specialized, relatively independent processors (2.2). Further, only one consistent message can be broadcast at a time, so that the mental news medium does not publish self contradictory information at any one time (2.1). And third, GW theory claims that there are contextual constraints on the conscious interpretation of the world, comparable to the editorial policies and practices of a newspaper that determine how it will select and interpret the news (4.0). In these ways the publicity metaphor seems helpful (Baars 1995: 177).

In this passage, Baars explores the features of similarity between the global workspace as a newspaper and the global workspace as mental activity embedded in neuronal processes (ERTAS). He then goes on with this metaphor of a newspaper and extends the GW model to handle how to interpet significant features of the news. This he does through developing the notions of information emerging from unconscious cultural contexts into consciousness. Near the end of his book, Baars deals with the dissimilarity of the parts of the basic metaphor. He carefully notes the limitations of using metaphors to express theories.

Likewise, we have made a great efiort in this book to present useful metaphors for our theory, such as the image of a conference of experts, each competing for access to a central blackboard. But every concrete metaphor is really inadequate. For example, the conference image fails to show that expert processors in GW theory are decomposable, while human experts are not (1.4.2). (Human experts have a tendency to stop running when they are decomposed.) This point is quite general: Metaphors are useful but ultimately inadequate representations of a more abstract and complex reality (Lakoff \& Johnson 1980). In science they must be used with care.

In general, an imageable metaphor seems to serve the function of evoking and recruiting conceptual processes that are more abstract and often more accurate than the image itself. These abstract entities may be impossible to experience qualitatively. Hence the need for visual figures, audible words, and concrete metaphors. These can be made qualitatively conscious when needed, to stand for abstract nonqualitative entities (Baars 1995: 285).

Baars extends the metaphor of Global Workspace into a Theater metaphor in his later work, The Theater of Consciousness, implying that the former can be contained in the concept of the later. 
This book is based on a framework for understanding the large domain of evidence bearing on our personal experience, called Global Workspace theory. GW theory presents a "theater model" in which consciousness requires a central workspace, much like the stage of a theater (Baars 1997: viii).

This second book does provide new empirical evidence from Positron Emission Tomography (PET) studies of cognitive activations in the brain supporting his account of consciousness as a theater. Baars also links working memory to the theater metaphor when he states: "Working memory is like a theater stage". (Baars 1997: 41 [Italics in the original]). How they are related is not precisely stated but he does further include within working memory the processes of innner speech and visual imagery.

As with the Global Workspace, Baars admits a metaphoric status to his enlarged Theater model, but he seem to claim more than a theoretic status for this metaphor when he states:

Scientific metaphors are scaffolding that help us to grasp and simplify complicated problems. But scafoldings are useless without real bricks and concrete, and these models are mere doodles on a napkin in the absence of a large constellation of specific research findings: studies of the cellular wiring of the visual cortex, for example, and careful analyses of the remarkable effects of selective brain damage, the role of perception in action control, a century of psychophysical studies, and research on memory, imagery, language, and much more...

Like any metaphor, the theater architecture is useful only up to a point, we will keep track of its flaws as well as its uses as we go along. Yet is does provide a useful starting place. In the following chapters we use it to think about a large body of evidence about consciousness. We will not treat the theater metaphor as theory, though all current integrative theories can be thought of as theaters. We will use it to simplify the evidence (Baars 1997: 54 [Italics added]).

Metaphors serve the role of allowing one to perceive some of the abstract qualities of a theory in concrete form. Baars leaves the question of the nature of the abstract forms underlying metaphors unanswered. Nor does he suggest why one metaphor is better than another as a basic metaphor presupposed by a theory, except that by indirect inference one must suppose that his choice of the theater metaphor fits the empirical data better than other metaphors. Finally, Baars does not explore the consequence that self awareness of the choice of a metaphor like theater implies a close tie between metaphors and consciousness itself. In Baar's view, the researcher necessarily consciously chooses a metaphor to express abstract representations that cannot be fully and consciously understood. He also seems to claim that because so much scientific evidence supports the theater metaphor, it is not a theory but rather can be used to "simplify" the data. But isn't that just what theories do: organize, simplify and suggest further questions? 
Daniel Dennett also consciously chooses among metaphors to explain consciousness (Dennett 1991). He argues that consciousness is not a mystery and can be explained. But his explanation depends upon replacing our traditional way of thinking about consciousness which he calls the Cartesian Theater by a counterintuitive view which he calls a virtual machine a form of software that constantly edits itself and produces Multiple Drafts of the contents of consciousness.

The level of description and explanation we need is analogous to (but not identical with) one of the "software level" of description of computers: what we need to understand is how human consciousness can be realized in the operation of a virtual machine created by memes in the brain. Here is the hypothesis I will defend: Human consciousness is itself a huge complex of memes (or more exactly, mem-effects in brains) that can best be understood as the operation of a "von Neumannesque" virtual machine implemented in the parallel architecture of a brain that was not designed for any such activities. The powers of this virtual machine vastly enhance the underlying powers of the organic hardware on which it runs, but at the same time many of its most curious features, and especially its limitations, can be explained as the byproducts of the kludges that make possible this curious but effective reuse of an existing organ for novel purposes (Dennett 1991:210).

Dennett's metaphor of the Cartesian Theater represents the modern assumption that a place exists in the brain to order and control conscious experience. It does not express dualism but continues as a remnant of the materialistic side of Descartes' dualism.

Let's call the idea of such a centered locus in the brain Cartesian materialism, since it's the view you arrive at when you discard Descartes' dualism but fail to discard the imagery of a central (but material) Theater where "it all comes together"....

The Cartesian theater is a metaphorical picture of how conscious experience must sit in the brain. It seems at first to be an innocent extrapolation of the familiar and undeniable fact that for everydary, macroscopic time intervals, we can indeed order events into the two categories "not yet observed" and "already observed" (Dennett 1991: 107).

Instead of the Cartesian Theater,Dennett envisions a virtual machine consisting of parallel brain proceses producing Multiple Drafts presenting conscious events. These Drafts are constantly edited and revised. Much of this activity is similar to the interactions between unconscious processes in the brain and unconscious contexts in culture described in Baar's GW theory. Although agreeing with Baars' metaphor of various experts contributing to a concensus view of events, even though he does not explicitly reject Baar's Global Workspace, by implication he must at least be suspicious of it because it serves as 
a coordinating place like the Cartesian Theater (Dennett 1991: 257ff.). But for all of Dennett's parallel processes and apparent chaotic temporal events, he faces the problem of how these come together into seemingly focused images experienced in perception. Dennett, however, leaves that problem for future exploration; he wants to demolish the remnants of the ghost-in-the-machine presupposition and replace it with consciousness-isa-distributed-brain-process akin to but not identical with the software operation of a computer.

In replacing the Cartesian Theater with Multiple Drafts of a Virtual Software Machine, Dennett acknowledges that he has replaced one metaphor with another rather than replacing a metaphoric theory with a non-metaphoric literal theory.

My explanation of consciousness is far from complete. One might even say that it was just a beginning, but it is a beginning, because it breaks the spell of the enchanted circle of ideas that made explaining consciousness impossible. I haven't replaced a metaphorical theory, the Cartesian Theater, with a nonmetaphorical ("literal, scientific") theory. All I have done, really, is to replace one family of metaphors and images with another, trading in the Theater, the Witness, the Central Meaner, the Figment, for Software, Virtual Machine, Multiple Drafts, a Pandemonium of Homonuculi. It's just a war of metaphors, you say - but metaphors are not "just" metaphors; metaphors are the tools of thought. No one can think about consciousness without them, so it is important to equip yourself with the best set of tools available. Look what we have built with our tools. Could you have Imagined it without them (Dennett 1991: 455)?

Presumably, Dennett thinkgs that his new Metaphor of a Virtual Software Machine explains the evidence of modern neuroscience better than the Cartesian Theater even though the new metaphor seems counterintuitive to the old one. In the passage above, however, Dennett introduces the problem of self reference when he asserts that "No one can think about consciousness without them [metaphors]". If to conceive of consciousness one must presume a metaphor and if one concedes that metaphors fall within the domain of consciousness, then metaphor itself becomes an unexplained but presumed form of consciousness reflecting upon consciousness. Reflexive relationships like the consciousness of consciousness - in this case metaphoric consciousness of consciousness - are notorious for introducing at least confusion if not outright paradox. One way out that Dennett seems to reject is to claim that there exists nonmetaphoric literal language based upon third person agreements about the perceptual world. One might further claim that consciousness of external third person events differs from metaphoric consciousness of consciousness presumed in an explanation about how the brain produces consciousness.

But Dennett's entire enterprise uses imagination in the form of thought experiments to develop his explanation of consciousness. In this, he seems even 
more firmly anchored in a world of competing imaginative metaphors. Why are some metaphors better than others? Because they are more coherent? Because they are developed from contemporary scientific evidence? Or because they are asserted by Dennett? Reasons for accepting some and rejecting other metaphors seems to vary throughout his discussion.

John Searle in his The Rediscovery of the Mind (Searle 1992) also seems to replace an old metaphor about consciousness - consciousness is mental - by a new metaphor consciousness is a biological feature of the brain. Searle states:

Consciousness, in short, is a biological feature of human and certain animal brains.

It is caused by neurobiological processes and is as much a part of the natural biological order as any other biological features such a photosynthesis, digestion, or mitosis. This principle is the first stage in understanding the place of consciousness within our world view. The thesis of this chapter so far has been that once you see that atomic and evolutionary theories are central to the contemporary scientific world view, then consciousness falls into place naturally as an evolved phenotypical trait of certain types of organisms with highly developed nervous systems (Searle 1992: 90).

Our traditional manner of speaking about mental events that cannot be physcial events stands in the way of our acceptance of the identification of consciousness as a bioilogical feature of the brain. Searle claims that consciousness exists as an intrinsic property that is both mental and at the same time physical. At times, Searle's descriptions of consciousness as a biological feature seem to imply that not only does consciousness stand as an intrinsic property but also as a literal one. This implication arises from his rejections of the "metaphors" of introspection and privileged access.

Philosophers have invented another metaphor for describing certain features of subjectivity that seems to me even more confused than the commonsense metaphor of introspection, and that is "privileged access". For the visual metaphor of introspection, we are tempted to substitute the spatial metaphor of privileged access, a model that suggests that consciousness is like a private room into which only we are allowed to enter. Only I can go inside the space of my own consciousness. But this metaphor doesn't work either, because for there to be something to which I have privileged access, I would have to be different from the space in which I enter. But just as the metaphor of introspection broke down when the only thing to be observed was the observing itself, so the metaphor of a private inner space breaks down when we understand that there isn't anything like a space into which I can enter, because I cannot make the necessary distinctions between the three elements of myself, the act of entering, and the space in which I am supposed to enter (Searle 1992: 98). 
Searle destroys these metaphors by demonstrating their impossibility when taken literally. If these metaphor were left as metaphors without claims to represent the reality of consciousness, they would remain hypothetical and acceptable. Searle explicitly recognizes the hypothetical value of metaphor in the following quotation.

There is nothing harmful, misleading, or philosophically mistaken about as-if metaphorical ascriptions. The only mistake is to take them literally (Searle 1992: 82).

This attitude towards metaphor and Searle's claim that consciousness is an intrinsic biological feature, might lead one to infer that Searle also should deny any metaphoric quality to his description of consciousness. Yet, he candidly admits that consciousness as a physical property differs importantly from other physical properties in that consciousness cannot be reduced to other phenomena. Although the appearances of physical phenomena can be reduced to physical realities, consciousness cannot be so reduced.

Consciousness fails to be reducible, not because of some mysterious feature, but simply because by definition it falls outside the pattern of reduction that we have chosen to use for pragmatic reasons. Pretheoretically, consciousness, like solidity, is a surface feature of certain physical systems. But unlike solidity, consciousness cannot be redefined in terms of an underlying microstructure, and the surface features then treated as mere effects of real consciousness, without losing the point of having the concept of consciousness in the first place (Searle 1992: 122-123).

At least in one respect, that of failing to be reducible, consciousness as a physical, biological feature differs as an exception from all other physical features. This would seem to suggest that notwithstanding Searle's denial of mystery, his concept of consciousness if not fully metaphoric, contains some tension between the referents of the major parts of his definition. This definition is at least an epiphor. Although Searle might like to keep his notion of consciousness as a literal statement, his inability to justify consciousness as a fully physical concept fails to avoid a metaphoric quality for consciousness. At one point, Searle suggests that the progress of neuroscience would eliminate the problem.

If we had an adequate science of the brain, an account of the brain that would give causal explanations of consciousness in all its forms and varieties, and if we overcame our conceptual mistakes, no mind-body problem would remain (Searle 1992: 100).

But the claim that future research will fully justify our present position could be just as readilty employed by a materialist or a dualist or an advocate of any position. In its present form, Searle's description of consciousness as a biological feature (with subjective mental qualities) of the brain does not escape a metaphoric status. 
Gerald Edelman has developed a biological theory of consciousness founded upon a basic metahor of evolution (Edelman 1989).

As a useful metaphor, I called this application to the nervous system of population Thinking (an approach invented by Darwin) "neural Darwinism”. Of course, I expected that the mechanisms by which diversity was generated and selection occurred in somatic neural function would be quite difeerent from those of evolution (Edelman 1989:39).

A Theory of Neuronal Group Selection (TNGS) forms the heart of this basic metaphor. TNGS relates perceptual categorization to neural functioning through developmental selection, experiential selection, and reentrant mapping. The latter, reentrant mapping, plays a crucial role in the development of consciousness.

Reentry is a proces of temporally ongoing parallel signalling between separate maps along ordered anatomical connections. Reentrant signaling can take place via reciprocal connections between maps (as seen in corticocortical, corticothalamic, and thalamocortical radiations); it can also occur via more complex arrangements such as connections among cortex, basal ganglia, and cerebellum (Edelman 1989:49).

Distinguishing consciousness into primary consciousnes and higher consciousness, Edelman describes the former as emerging from memory that relates present value-free perceptions to previous value-laden perceptions. And this emergence of primary consciousness has an evolutionary basis.

The main proposal is that primary consciousness arose as a result of two novel evolutionary events. (1) The first event led to the development of special memory repertoires composed of neuronal groups specifically dedicated to storing past matchings of value to perceptual categories... (2) The second evolutionary event led to the development of new circuits allowing reentrant signaling between these special memory repertoires and those currently devoted to sensorimotor sampling of the environment for perceptual categorization (Edelman 1989: 95-96).

Primary consciousness, then arose to relate earlier value-laden perceptions to present perceptions. This relationing function takes place in memory where recategorization takes place. Two neural systems are thus mediated: (1) the categorization of interoceptive inputautonomic, hypothalamic, and endocrine mediated by limbic and brain-stem circuits; and (2) the categorization of exteroceptive input-peripheral, voluntary motor, proprioceptive, and polymodal sensory signals mediated by thalamus and cortical areas. The comparison of these two systems (primary consciousness in memory) takes place at the hippocampus, septum and cingulate gyri. In this description, Edelman carefully locates primary consciousness in well established neuronal systems. He also roots his notions in an evolutionary context through the use of his metaphor that "neuronal groups 
select" (in an evolutionary sense) neural proceses that produce primary consciousness.

Higher consciousness also develops through TNGS to fulfill the need for future plans. Through the development of symbols, especially language, the brain develops the ability to be conscious of the past, present and future and to be conscious of consciousness. These are the forms of higher consciousness.

Like Baars, Edelman has developed a concept of consciousness based upon a metaphorically driven theory rather than using a metaphoric definition of consciousness as did both Dennett and Searle All of these authors, however, explicitly admit their adoption of metaphors. Is this merely an historical accident or a useful pragmatic move? Or are metaphors a necessary feature for any description or theory of metaphor? What would literal statements about consciousness look like?

\section{The possibility of mathematical models which contain literal statements about consciousness}

Lakoff and Johnson claim in their Metaphors We Live By that all language is metaphoric with the consequence that no literal language exists (Lakoff and Johnson, 1980). If this were true then searching for any literal statements about consciousness would be impossible. But literal statements do exist in ordinary language where we make commonsense statements about ourselves and our environment. Metaphors are formed by juxtaposing ordinary literal linguistic concepts in unusual ways thereby suggesting possible new meanings. Some of these vibrant, extremely suggestive, possibile metaphors remain conjectures. Others become more expressive of experience and become commonly used and, therefore, no longer metaphoric but ordinary and literal. Language often flows from the ordinary and literal to the metaphoric and then back again to the ordinary through the creation of newly accepted meanings (Mac Cormac, 1985). The very recognition of a metaphor as metaphoric depends upon the contrast of the literal usually conveyed by ordinary language spoken or written in a commonsense manner.

Even if there are literal statements, however, what would literal statements about consciousness look like? Literal statements about the environment can be shared through expressions of language. By contrast, statements about the contents of consciousness cannot be shared in the same way. We can agree that we experience consciousness but there exists no direct human perception through the senses to agree upon the contents of consciousness. This fact of the privacy of consciousness led some philosophers and some psychologists earlier in the century to deny the very existence of both minds and consciousness (Ryle, 1949). 
Behaviorism sought to construct a psychology founded upon the bedrock of direct empirical observations thereby relegating mind and consciousness to the status of unconfirmable, theoretical constructs. But the commonsense experience of thinking and self awareness seemed to many directly contradictory to these behavioral assumptions. Even though one could not give a directly perceivable conscious experience to others, conscious thoughts could be shared through language. In the latter half of the twentieth century, mind and the mental returned to the fields of philosophy and psychology as legitimate objects of study. We have already noted that researchers from these two fields joined neuroscientists to create the new discipline of cognitive studies.

Gilbert Ryle denigrated mind as the "ghost in the machine". The advent of new imaging procedures, however, has enabled scientists directly to explore that machine, the brain, more carefully searching for the neuronal processes which generate both thinking and consciousness presenting evidence which can be publicly confirmed (Mac Cormac 1995). This evidence consists of increased metabolism or increased blood flow to particular regions of the brain. We noted above that both Baars and Edelman attempted to locate the origins of consciousness in brain activity. Baars found it in the extended reticular-thalamic activity system (ERTAS). Edelman's theory of neuronal group selection (TNGS) is a brain process of the alteration of present neural connections by memory of past connections occasioned by perceptions (reentrant mapping). Yet neither scientist could present literal statements about consciousness, their accounts remained metaphoric and suggestive. In Baars' new book In The Theater of Consciousness he does recognize positron emission tomography (PET) scans of the brain as empirical tests of consciousness (Baars, 1997: 12,19).

PET and functional Magnetic Resonance Imaging (fMRI) offer the possibility as Baars acknowledges of an empirical investigation of the origins of both mind and consciousness in brain processes. PET and MRI studies of cognitive functions have investigated perceptual and semantic processes which while sometimes requiring awareness have not been designed in such a way that one could isolate consciousness as a distinct process. It is very likely that consciousness like memory occurs in no single region of the brain - there is probably not a brain region which serves as the origin of consciousness. Memory is spread throughout the brain. By contrast, vision can be identified in the rear (occipital region) of the brain in the visual cortex. Identification of objects or words by sight, however, involves more than just the visual cortex as recall and semantic processing take place elsewhere. The brain is an incredibily complex organ with a multitude of connections among millions of neurons firning rapidly in ever changing patterns.

The brain can be represented as a electrical-chemical complexity of relationships where impulses of energy are rapidly communicated from one part of many other parts. Ionic exchanges across membranes are triggered by chemical 
neurotransmitters; neurons are both excited and inhibited by these impulses rapidly conveyed across membranes. This complex activity seems to be nonlinear with stable patterns emerging from chaos.

PET can be used to study cognitive behavior by displaying increased metabolism in neurons (when flurodeoxygloucose [FDG] is used as the radionuclide). Neurons activated by cognitive challenges are presumed to have an increased metabolism. The temporal resolution of FDG is so long as to make studies of cognition difficult. With a much better temporal resolution, Oxygen- 15 with a half life of 123 seconds, is usually the choice for cognitive studies. These studies display increased blood flow rather than increases in metabolism (the relationship between metabolism and blood flow can be represented by a nonlinear algorithm). Activated neurons demand more oxygen and therefore greater blood flow.

Areas of the brain with increased blood flow during cognitive activations vary both spatially and temporally. Spatial variations can be described by PET scans but temporal sequences of the activation of different areas thus far cannot be described by PET. A simultaneous use of MRI and PET or Electroencephalographs (EEG) and PET might provide better temporal resolution. The latter combination is probably more feasible technically than the former since the apparatus for visual stimulation (usually a Cathode Ray Tube (CRT)) would interfere with MRI.

If one were looking for a literal statement describing a brain process involving consciousness, what would it look like? One possibility might be a mathematical algorithm describing both the spatial and temporal aspects of areas of the brain activated (measured by either increased metabolism or increased blood flow - increased electrical activity for the EEG if used in consort with PET) during a cognitive challenge. But what kind of algorithm would one select to represent this process?

The spatio-temporal process seems to be a nonlinear dynamical system of self organization. Skarda and Freeman assert:

Perception is a self organized dynamic process of interchange inaugurated by the brain in which the brain fails to respond to irrevelant input, opens itself to the input it accepts, reorganizes itself, and then reaches out to change its input. We suggest that the self organized process that replaces environmental input with an internally generated, chaotic activity pattern is one that gives "biological meaning" to the stimulus (Skarda \& Freeman 1990:279).

Mathematical chaos theory does not mean random activity but rather activity which can be represented by an algorithm and under certain conditions seems random and under other conditions seems stable. The randomness or stability depends upon the values used to satisfy the algorithm which are not predictable beforehand. The chaotic algorithm under some values produces random behavior while under other 
values, the same algorithm produces stable patterns. The brain exhibits exactly this behavior, neurons fire randomly and then under certain conditions (perhaps those of perception and cognition) the firing neurons produce stable patterns.

The stable patterns of mathematical chaos are fractals, a form of geometry described by Benoit Mandelbrot (Mandelbrot, 1983). The fractal dimensions of these algorithms need not necessarily be integers, they can be fractions. Mandelbrot contends that fractal geometry better represents actual characteristics of the physical world than does Euclidean geometry. Others have employed fractals to model biological mechanisms like brain processes (Mac Cormac \& Stamenov, 1996; West et al, 1995).

Although not all chaotic algorithms are fractals, all of the stable attractors of chaotic algorithms seem to be fractals. Hence, if one could find the chaotic algorithms of brain activity, the stable patterns during consiousness or mental activity (incluidng preception) would be fractal. Starting the other way round, if one could capture the stable patterns of brain activity (spatial and temporal) by means of PET and/or combined PET/EEG scans during cognitive activations, one might discover the fractal patterns and from those construct the chaotic algorithms. We have done just that in discovering the spatial fractals including fractal dimension for the recognition of geometric figures (Mac Cormac \& McKinney, 1998). Now we must discover the temporal fractals for these same cognitive activations therey allowing use to construct nonlinear dynamic mathematical algorithms which capture both the spatial and the temporal aspects of neuronal processes.

Here then in the nonlinear dynamic systems (mathematical chaos) descriptive of neuronal processes under cognitive activation, we find literal statements of consciousness and cognition. The fractal aspects of these systems (algorithms) are like the object words of empirical sentences which refer to intersubjectively perceiveable things and/or experiences in the physical world. Are metaphors necessary for descriptions of how consciousness and thinking emerge from the brain? If the program now partially fulfilled succeeds, the answer to this question will be -no, metaphors are useful but not necessary since literal statements about brain processes which generate consciousness can be presented in the form of chaotic (nonlinear dynamical) mathematical systems discoverable through a combination of PET-EEG experiments. 


\section{Acknowledgement}

Part of "Metaphoric descriptions of consciousness" was published in The Noetic Journal Volume 2, No. 1 (January 1999) and is reprinted here with permission.

\section{References}

Baars, B. J. 1995, A Cognitive Theory of Consciousness. Cambridge: Cambridge University Press.

Baars. B. J. 1997, In The Theater of Consciousness. New York: Oxford University Press. Dennett, D. C. 1991, Consciousness Explained. Boston: Little Brown and Co.

Edelman, G. M. 1987, Neural Darwinism: The Theory of Neuronal Group Selection. New York: Basic Books.

Edelman, G. M. 1989, The Remembered Present: A Biological Theory of Consciousness. New York: Basic Books.

Lakoff, G. \& Johnson, M. 1980, Metaphors We Live By. Chicago: University of Chicago Press.

Mac Cormac, E. R. 1985, 1989, A Cognitive Theory of Metaphor. Cambridge, Mass.: MIT Press [Bradford Books].

Mac Cormac, E. R.1995, Die Geographic und Geometrie des Gehirns: Modifikation unserer Begriffvon Geist und Bewusstsein, in Lenk, H. \& Poser, H. (eds.), Neue RealitatenHerausforderung der Philosophic. Berlin: Akademie Verlag.

Mac Cormac, E. R. 1996, Fractal thinking: self organizing brain processing, in Mac Cormac,

E.R. \& Stamenov, M. I. (eds.), Fractal Brain. Fractal Mind: In Search of a Symmetry Bond. Amsterdam and Philadelphia: John Benjamins.

Mac Cormac, E. R. \& McKinney, C. J. 1998, Chaos: a mathematics for heart and mind. North Carolina Medical Journal 59:3 (May/June 1998) 161-165.

Mandelbrot, B. B. 1983, The Fractal Geometry of Nature. New York: W. H. Freeman. Ryle, G. 1949, The Concept of Mind. Chicago: University of Chicago Press.

Searle, J. R. 1992, The Rediscovery of the Mind. Cambridge, Mass.: MIT Press [Bradford Books],

Skarda, C. A. \& Freeman, W. J. 1990, Chaos and the new science of the brain. Concepts of Neuroscience 1:2, 275-285.

West, B. J., Marcos, N. N. \& Voyko, K. 1996, Fractal probablility density and EEG/ERP time series, in Iannaccone, P.M. \& Khokha, M. (eds.), Fractal Geometry in Biological Systems. Boca Raton: CRC Press. 